\title{
POLÍTICAS LINGÜÍSTICAS Y ENSEÑANZA DE LENGUAS: APROXIMACIONES AL PROGRAMA “CENTRO DE LENGUAS EXTRANJERAS"
}

\author{
Ana Leticia Andregnette Roscigno \\ Máster en Formación de Profesores de Español \\ como Lengua Extranjera \\ Contacto: analeticia.andregnette@gmail.com \\ ORCID: 0000-0002-0358-9768
}

Recibido: 16 de noviembre de 2019

Aceptado: 16 de diciembre de 2019

DOI: $10.25087 /$ resur8a2

\begin{abstract}
Resumen
El Estado uruguayo siempre ha considerado beneficiosa la enseñanza de lenguas modernas en educación secundaria. El francés, el inglés y el italiano fueron las lenguas que tradicionalmente integraron los distintos planes de estudio. El portugués, por motivos político-lingüísticos, nunca estuvo incluido. En 1995, se estableció que el inglés fuera la única lengua extranjera de enseñanza obligatoria en la educación media -pública y privada. En 1996, se creó el Programa "Centro de Lenguas Extranjeras" (CLE) para ofrecer, a alumnos del ámbito público, cursos opcionales y extracurriculares de francés, italiano y portugués incorporándose, posteriormente, el alemán y la lengua de señas uruguaya. El objetivo de la ponencia ${ }^{1}$ es presentar una investigación realizada sobre el Programa CLE. Al comienzo del trabajo, se desarrollan brevemente algunas las medidas tomadas por el Estado con relación a la enseñanza de lenguas extranjeras en los siglos XIX, XX y XXI. Seguidamente, se analiza la fundamentación de la creación del Programa, sus características y su evolución en términos estadísticos. La investigación muestra que el número total de estudiantes del Programa, por un lado, nunca ha llegado al 10\% del público al que
\end{abstract}

\footnotetext{
${ }^{1}$ Una versión de este artículo se presentó como ponencia en las Jornadas 2017 -Profesor W. BenavidezHumanidades digitales y abiertas. VII Jornadas de Investigación, VI Jornadas de Extensión y V Encuentro de Egresados y Maestrandos. Facultad de Humanidades y Ciencias de la Educación, en octubre de 2017, Montevideo.
} 
está dirigido y, por otro que, entre 2002 y 2015, ese número ha descendido de manera constante. Asimismo, se observa que, si bien el portugués figura como la lengua con mayor cantidad alumnado seguida por el italiano, el francés, y el alemán, la limitada información disponible obliga a relativizar las conclusiones. Finalmente, la investigación deja planteadas nuevas interrogantes: por un lado, con respecto a la coherencia entre el discurso estatal y la realidad del Programa CLE y, por otro, sobre las causas de la escasa información disponible acerca del mismo.

Palabras Clave: políticas lingüísticas; enseñanza lenguas; centro lenguas extranjeras

\title{
POLÍTICAS LINGUÍSTICAS E ENSINO DE LÍNGUAS: APROXIMAÇÕES AO PROGRAMA “CENTRO DE LÍNGUAS ESTRANGEIRAS”
}

\section{Resumo}

O Estado uruguaio sempre considerou benéfico o ensino de línguas modernas no ensino médio. O francês, o inglês e o italiano foram as línguas que tradicionalmente integraram os diferentes planos de estudo. O português, por razões político-linguísticas, nunca foi incluído. Em 1995, foi estabelecido que o inglês fosse a única língua estrangeira obrigatória no ensino médio - público e privado -. Em 1996, foi criado o Programa "Centro de Línguas Estrangeiras” (CLE) para oferecer, a alunos do âmbito público, cursos opcionais e extracurriculares de francês, italiano e português incorporando-se, posteriormente, o alemão e a língua de sinais uruguaia. O objetivo da exposição é apresentar uma investigação sobre o programa CLE. No início do trabalho, desenvolvem-se brevemente algumas das medidas tomadas pelo Estado em relação ao ensino de línguas estrangeiras nos séculos XIX, XX e XXI. Seguidamente, analisase o fundamento da criação do Programa, suas características e sua evolução em termos estatísticos. A investigação mostra que o número total de alunos do Programa, por um lado, nunca atingiu 10\% do público a que se destina e, por outro lado que, entre 2002 e 2015, esse número diminuiu constantemente. Também se observa que, embora o português figure como a língua com maior número de alunos, seguida pelo italiano, o francês, e o alemão, a limitada informação disponível obriga a relativizar as conclusões. Por último, a investigação levanta novas questões: por um lado, quanto à coerência entre o discurso estatal e a realidade do Programa CLE e, por outro, sobre as causas da escassa informação disponível sobre este.

Palavras chave: Políticas de linguagem; Ensino de línguas; Centro de línguas estrangeiras

\section{LANGUAGE POLICIES AND LANGUAGE TEACHING: APPROACHES TO THE PROGRAMME "FOREIGN LANGUAGE CENTRE”}

\begin{abstract}
The Uruguayan State has always considered the teaching of modern languages in secondary education beneficial. French, English and Italian were the languages that traditionally integrated the different curricula. Portuguese, for political-linguistic reasons, was never included. In 1995, English was established
\end{abstract}


as the only foreign language of compulsory education in public and private secondary education. In 1996, the Centre for Foreign Languages (CLE) Programme was set up to offer optional and extra-curricular courses in French, Italian and Portuguese to students from the public education. Later, German, and Uruguayan Sign Language were added. The objective of the paper is to present an investigation carried out on the CLE Programmme. To begin, some of the measures taken by the State in relation to the teaching of foreign languages in the nineteenth, twentieth and twenty-first centuries are briefly developed. Then, the basis of the creation of the Programme, its characteristics and its evolution in statistical terms are analyzed. The research shows that the total number of students in the Programme has never reached 10\% of the public it is aimed at, and that, between 2002 and 2015, that number has steadily declined. It is also noticed that, although Portuguese is the language with the highest number of pupils, followed by Italian, French and German, the limited information available makes it necessary to relativise the conclusions. Finally, the research raises new questions: on the one hand, regarding the coherence between the state discourse and the reality of the CLE Programme and, on the other, about the causes of the limited information available about it.

Keywords: Language policies; Teaching languages; Foreign language Centre

\section{Introducción}

Las políticas y planificación lingüísticas llevadas adelante por el Estado uruguayo, principalmente desde 1877, han respondido a múltiples factores, y han recaído en distintos ámbitos de las políticas educativas (Anep, 2008a; Brovetto, 2010; Barrios, 2013; Oroño, 2016).

Considerando que el Uruguay ha tenido una larga tradición en la enseñanza de lenguas extranjeras, en el marco del seminario Discursos hegemónicos, representaciones y prácticas lingüísticas de la Licenciatura de Lingüística, a cargo de la Prof. Dra. Graciela Barrios, se realizó una investigación que analiza el Programa "Centros de Lenguas Extranjeras" (en adelante Programa $C L E$ ), en tanto forma parte de las políticas y planificación lingüísticas implementadas a partir de la última década del siglo XX. En esta ponencia se presentan los aspectos sustanciales del referido trabajo, actualizado con la nueva información disponible.

Con respecto a los aspectos metodológicos, la investigación estuvo basada en un análisis bibliográfico y documental. La primera etapa consistió en el relevamiento de la información vinculada a la enseñanza de lenguas extranjeras en la educación uruguaya, y al Programa $C L E$ desde el momento de su creación hasta la actualidad. La búsqueda se centró en artículos de investigadores y en información publicada por organismos oficiales (informes, anuarios, resoluciones, etc.) con todo lo cual se formó un corpus que fue la base del análisis. Durante el trabajo se enfrentaron tres tipos de dificultades: en primer lugar, la escasa información dispo- 
nible, en segundo lugar, que dicha información se encontró fragmentada y dispersa en distintas fuentes y, en tercer lugar, que, en algunas ocasiones, distintas fuentes ofrecieron la misma información con datos diferentes o contradictorios.

A continuación, se desarrollará brevemente la evolución de la enseñanza de las lenguas extranjeras en la educación media hasta la creación del Programa CLE y, seguidamente, se analizará la fundamentación de la creación del Programa, su situación actual y su evolución en términos estadísticos.

\section{Políticas lingüísticas y enseñanza de lenguas extranjeras en Uruguay: Anteceden- tes históricos}

Barrios (1996) señala que, para comprender las políticas lingüísticas llevadas adelante por el Estado uruguayo, hay que tener presente su surgimiento como Estado independiente, el cual, no fue resultado de la voluntad unánime de su pueblo, ni de un sentimiento de nación que identificara a sus habitantes. Este hecho le llevó a que, en sus primeras décadas, debiera enfrentar, desde el punto de vista político, diferentes escenarios que, agravados por guerras civiles y una economía débil, hicieron aún más difícil su organización y desenvolvimiento, hasta ya avanzado el siglo XIX (Traversoni, 1987; Nahum, 1994; Barrios, 2011, 2013, 2015).

Por otra parte, desde el punto de vista sociocultural, en el país se podían distinguir dos regiones: la región norte-noreste, con un sustrato lingüístico portugués; y la región centro-sur y litoral-oeste, con una base lingüística hispánica, a la que se sumó la gran influencia de inmigrantes europeos fundamentalmente de origen italiano, que llegaron durante la segunda mitad del siglo XIX y primera mitad del XX (Barrios, 1996). La diversidad lingüística, social y cultural entre las dos regiones era producto de sus diferentes tradiciones, la cual, incrementada por el empuje migratorio que desde 1835 tenía el país, hizo manifiesta la necesidad de un sistema educativo con una lengua única que funcionara como símbolo de identidad nacional (Behares y Brovetto, 2009; Bralich, 2013). Una de las medidas más importantes fue la reforma educativa instrumentada a través del Decreto-Ley de Educación Común -No. 1.350- de 1877 (Barrios, 1996, 2015; Oroño, 2011). Dicha norma instrumentó la obligatoriedad de la educación primaria y formalizó el español como única lengua de instrucción en todo el territorio nacional (Oroño, 2016). La disposición tenía como objetivo que el español funcionara como símbolo de identidad, pero también buscaba la consolidación de las fronteras culturales y la defensa de la soberanía del Estado. La presencia del portugués en la zona de frontera con el Brasil era tenida 
como una amenaza no solo económica y política sino también cultural, de aquí que no se buscara alcanzar solamente la unificación lingüística, sino también la desaparición de las lenguas regionales y minoritarias que existían en el territorio (Barrios, 1996, 2013, 2015; Oroño, 2011, 2012, 2016).

\section{Enseñanza de lenguas extranjeras en la Educación media: antecedentes y la crea- ción del Programa CLE. La situación actual}

La sociedad uruguaya, al iniciarse en la vida independiente, carecía de instituciones de enseñanza que medianamente pudieran satisfacer sus necesidades. La inestabilidad política hacía difícil organizar la educación, no obstante, se dictaron disposiciones que pretendieron impulsar el sistema educativo (Bralich, 2013). La enseñanza secundaria tenía limitado desarrollo; se restringía al ámbito privado y se caracterizaba por tener problemas económicos y de dirección (MEC, 2014). Recién fue implementada de manera sistemática hacia la mitad del siglo XIX, aunque "se cuenta con información administrativa confiable [...] a partir de 1885" (MEC, 2014: 79).

Con respecto a la enseñanza de lenguas extranjeras, a pesar de la escasa información, se encuentran registros que muestran su presencia en los planes de estudio de algunas instituciones. Hacia 1830, la Escuela Especial de Comercio (posteriormente Escuela Mercantil) tenía entre sus asignaturas la enseñanza de francés (Araujo,1906). Unos años más tarde, el Colegio de los Padres Escolapios, ofrecía el estudio de francés y de italiano, mientras que el Colegio Oriental de Humanidades, además de estas dos lenguas, incluía también la enseñanza de inglés (Reyes Abadie y Vázquez Romero, 2000a; Traversoni,1987).

En 1849, cumpliendo con lo establecido en disposiciones de 1833 y 1838, se inauguró la Universidad de la República, que tendría bajo su órbita toda la enseñanza pública: Enseñanza Primaria, Enseñanza Secundaria y Enseñanza Científica-Profesional (Reyes Abadie y Vázquez Romero, 2000b; ANEP, 2008b; MEC, 2014). La Enseñanza Secundaria, en ese momento, incluía francés e inglés entre sus materias de estudio (ANEP, 2008b; Ardao, 1950, citado en MEC, 2014). ${ }^{2}$

En 1877, se promulgó el Decreto-Ley de Educación Común -No. $1.350-{ }^{3}$ que, como se dijo anteriormente, organizó la educación primaria en todo el país y estableció que, en todas

\footnotetext{
${ }^{2}$ Sin embargo, Reyes Abadie y Vázquez Romero (2000a) señalan que estas asignaturas fueron incorporadas recién en 1860 .

${ }^{3}$ A partir de entonces, la Enseñanza Primaria salió de la órbita de la Universidad y pasó a depender
} 
las escuelas públicas, la enseñanza se impartiera en español con lo cual excluyó la educación en cualquier otra lengua (Behares y Brovetto, 2009). Con respecto a la enseñanza de lenguas extranjeras, la norma no hace ninguna mención.

En 1885, fue sancionada la Ley Orgánica de la Universidad de la República que mantuvo a la Enseñanza Secundaria dentro de su dominio, junto a la Enseñanza Científica-Profesional. En aquel momento, los estudios en la enseñanza secundaria se desarrollaban a lo largo de seis años. En el plan de estudios de 1887, el inglés y el francés estaban entre sus asignaturas (ANEP, 2008b).

En 1912, la Ley $N^{\circ} 3.939$ creó los Liceos Departamentales. Su plan curricular fue similar al de los Liceos de la Capital por lo cual también estaba presente la enseñanza de inglés y de francés (ANEP, 2008b). En 1935, la Ley N 9.523 convirtió a la Enseñanza Secundaria en Ente Autónomo del Estado, con lo cual se independizó definitivamente de la Universidad (ANEP, 2008b; MEC, 2014).

Los posteriores planes de estudio (1941, 1963, 1968, 1976, 1986 y 1993), permitieron que, al terminar los seis años de educación secundaria, los alumnos hubieran accedido -según la orientación elegida en Bachillerato ${ }^{4}$ - a la enseñanza de dos o tres lenguas extranjeras: inglés y francés, o bien, inglés, francés e italiano. Los planes 76 y 86 implementaron la posibilidad de optar entre inglés o francés en Ciclo Básico y Bachillerato; y el plan $93^{5}$, entre francés, inglés o italiano a partir de $4^{\circ}$ año (cfr. La Paz Barbarich, 2012). El portugués nunca estuvo incluido en los planes de estudio.

Los argumentos que fundamentaron la enseñanza de estas tres lenguas fueron "argumentos de tipo instrumental" (Barrios, 1996:98), basados en una concepción de lengua formadora del pensamiento y medio para acceder a otras culturas (Gabbiani,1995 citado en Bertolotti, 2003). El aprendizaje de francés se justificó en "la influencia cultural de esa nación”, el de inglés en "su relevancia comercial" y el de italiano en "su importancia en el derecho y la medicina" (Bertolotti, 2003:78). Sin embargo, nunca se utilizó como argumento el gran porcentaje de población inmigrante de origen europeo - fundamentalmente italiano- que tenía la sociedad

exclusivamente de la Dirección General de Instrucción Pública, órgano creado por el mismo Decreto-Ley.

${ }^{4}$ La organización de la enseñanza secundaria en seis años divididos en dos etapas: Primer Ciclo o Ciclo Básico y Segundo Ciclo o Bachillerato, ha estado vigente con distintas denominaciones y diseños curriculares desde 1908 (Anep, 2008b).

${ }^{5}$ Microexperiencia que estuvo en funcionamiento en ocho liceos y luego en siete: tres en Montevideo y cuatro en el Interior (cfr. La Paz Barbarich, 2012). 
uruguaya (Bertolotti, 2003), como así tampoco se ha hecho referencia "a las tradiciones étnicas de grupos minoritarios" (Barrios, 1996:98).

El 7 de noviembre de 1995, en el Acta №57- Resolución N77-, el Consejo Directivo Central -CODICEN- de la Administración Nacional de Educación Pública -ANEP- resolvió que, a partir del año siguiente, el inglés fuera la única lengua extranjera de enseñanza obligatoria para todos los alumnos que ingresaran a Ciclo Básico (cfr. La Paz Barbarich, 2012).

Posteriormente, el 7 de marzo de 1996, el mismo organismo creó el Programa "Centros de Lenguas Extranjeras" para ofrecer la enseñanza de francés, italiano y portugués, de manera opcional y extracurricular, a estudiantes de los tres primeros años de Educación Secundaria y Educación Técnico Profesional (Acta $\mathrm{N}^{\circ} 8$ - Resolución $\left.\mathrm{N}^{\circ} 54\right)^{6}$.

Actualmente en Enseñanza Secundaria está vigente el plan 2006, donde el inglés es la única lengua extranjera presente tanto en Ciclo Básico como en Bachillerato. No obstante, hay planes de estudio que incluyen la enseñanza obligatoria de otra lengua además de inglés: el plan 1996EE dirigido a estudiantes con circunstancias especiales como extraedad o trabajo, ofrece la posibilidad de elegir entre inglés y francés en tercer año de Ciclo Básico y primero de Bachillerato; y el plan 1994 -Martha Averbug- que, desde 2012, se ha extendido a todos los cursos nocturnos de Bachillerato para Adultos y Jóvenes Extraedad y/o con condicionamientos laborales, tiene italiano en $5^{\circ}$ Humanístico y $6^{\circ}$ opción Derecho, además de inglés -única lengua extranjera ofrecida en las demás orientaciones-. Por su parte, en la Educación Técnico Profesional -UTU-, en el área de Educación Media Profesional, en los programas de Gastronomía figuran francés y/o portugués como asignaturas obligatorias.

\section{La integración regional y la enseñanza del portugués en el contexto del MERCO-} SUR.

El Tratado de Asunción, firmado entre Argentina, Brasil, Paraguay y Uruguay, en 1991, dio origen al Mercado Común del Sur -MERCOSUR-. Su objetivo principal fue crear oportunidades comerciales y de inversión para los países miembros. Sin embargo, en el Protocolo de Intenciones, firmado ese mismo año, también se declaró de interés difundir el aprendizaje de

\footnotetext{
${ }^{6}$ La Educación Técnico Profesional se encuentra en la órbita de la Administración Nacional de Educación Pública (ANEP), de aquí que el Consejo Directivo Central -CODICEN -órgano rector del ente- tenga la potestad de incluir a los alumnos pertenecientes al Consejo de Educación Técnico-Profesional (UTU) (CETP) en la citada resolución.
} 
los idiomas oficiales del Mercosur - español y portugués- con el propósito de promover un mayor desarrollo cultural, científico y tecnológico (Mercosur, 1991; Barrios, 1996).

De esta manera, a diferencia de lo que había ocurrido hasta ese momento, Estado uruguayo asumió el compromiso de trabajar en la construcción de una identidad regional, con lo cual, las medidas que habían buscado excluir la enseñanza del portugués de la educación obligatoria debieron comenzar a ser revisadas (Barrios, 1996).

\section{Fundamentación de la creación del Programa "Centro de Lenguas Extranjeras}

Como se dijo anteriormente, el Programa CLE fue creado con el objetivo de brindar a los alumnos de Ciclo Básico de Educación Secundaria y de Educación Técnica y Profesional (UTU), la enseñanza de francés, italiano y portugués, de manera opcional y extracurricular. Los fundamentos ofrecidos por el CODICEN fueron los siguientes:

I) que es tradicional en nuestro país la enseñanza de la lengua francesa y del italiano; II) que la creación del MERCOSUR y la importancia de Brasil en el mundo científico, tecnológico y artístico justifica la enseñanza asimismo del idioma portugués (Acta 8, Resolución 54, 7/3/96) (Bertolotti, 2003:82)

Las argumentaciones demuestran un cambio en la línea político-lingüística seguida hasta el momento por el Estado uruguayo. En el caso del francés y del italiano, no se hace referencia a las ventajas que puede tener su estudio en el desarrollo cognitivo de la persona, o en el ámbito laboral, o a su relevancia cultural debido al gran porcentaje de población de origen europeo en nuestra sociedad. La razón se encuentra solamente en que su enseñanza ha sido costumbre en nuestro país. Con respecto al portugués, se alega no solo la firma del Tratado de Asunción por el cual los Estados se obligaron a promover la integración regional, sino también a la importancia que Brasil ha adquirido a nivel internacional, la cual ha otorgado al portugués un prestigio cultural que valoriza su aprendizaje. De esta manera, el Estado uruguayo, que había estado "más o menos explícitamente durante décadas contra el portugués" (Barrios, 1996:97), abandona su postura tradicional y justifica su enseñanza.

\section{Hacia el "Proyecto definitivo de institucionalización". Las políticas y planifica- ción sobre lenguas extranjeras desde 2006}

En 2006, las cuestiones político-lingüísticas tomaron una relevancia particular al crearse, dentro del ámbito de la ANEP, la Comisión de Políticas Lingüísticas en la Educación Pública. 
En 2008, a través del Acta №37 -Resolución N¹14- se creó el Programa de Políticas Lingüísticas a los efectos de llevar adelante las propuestas de la Comisión.

Como primer paso se formuló la Misión y Visión de la ANEP en lo que respecta a las lenguas extranjeras:

La MISIÓN de la ANEP en materia de lenguas extranjeras es formar ciudadanos plurilingües que puedan, por medio del uso de las lenguas, interactuar en ámbitos sociales, académicos y/o laborales. Para alcanzar la calidad de plurilingües los ciudadanos conocerán una lengua extranjera de comunicación internacional (inglés), una lengua de comunicación regional (portugués) y una tercera lengua extranjera vinculada a sus intereses personales (italiano, francés, alemán u otras).

[...] La visión que este Grupo de Trabajo en Políticas Lingüísticas propone para la ANEP en materia de enseñanza de lenguas es la siguiente:

En el año 2030, todos los ciudadanos uruguayos que transiten o hayan transitado por la Educación Pública, en cualquiera de sus modalidades podrán comunicarse por medio de tres lenguas:

1. Podrán comunicarse en Inglés oral y escrito en calidad de usuarios competentes (Nivel B2). La enseñanza de Inglés se extenderá durante todo el ciclo de educación básica (Inicial 5, Primaria y Media).

2. Podrán comunicarse en Portugués oral y escrito en un nivel de usuario intermedio (Nivel B1), luego de haber tomado cursos de Portugués durante 3 años (final del ciclo de Educación Primaria y comienzo de Enseñanza Media).

3. Podrán comunicarse en un nivel de usuario intermedio en una tercera lengua a elección (Nivel B1), por la cual habrán optado durante la Enseñanza Media y la que habrán cursado por tres años. (Brovetto, 2010: 246-247)

En lo que tiene que ver con la Misión, la ANEP busca formar a los ciudadanos en lenguas extranjeras, a los efectos de que puedan alcanzar la calidad de plurilingües, entendiéndose por tales aquellos individuos que, teniendo como base su primera lengua, adquieren conocimientos en dos o más lenguas y pueden interactuar a través de ellas (Moreno, 2012).

En este sentido, su meta (la Visión) será lograr que, en el año 2030, todos los ciudadanos uruguayos, que hayan tenido su formación en la Educación Pública, sean capaces de comunicarse, tanto en forma oral como escrita, en tres lenguas: en primer lugar, en inglés en calidad de usuarios competentes (B2); en segundo lugar, en portugués en un nivel de usuario intermedio (B1) y, por último, en una tercera lengua a su elección, también en un nivel de usuario intermedio (B1).

En 2008, se promulgó la ley No. 18.437 - Ley General de Educación - que, en su artículo 40, literal I, numeral 5, establece: 
La educación lingüística tendrá como propósito el desarrollo de las competencias comunicativas de las personas, el dominio de la lengua escrita, el respeto de las variedades lingüísticas, la reflexión sobre la lengua, la consideración de las diferentes lenguas maternas existentes en el país (español del Uruguay, portugués del Uruguay, lengua de señas uruguaya) y la formación plurilingüe a través de la enseñanza de segundas lenguas y lenguas extranjeras.

A diferencia de los objetivos político-lingüísticos del Estado uruguayo en el siglo XIX, en el siglo XXI, se propone el respeto, no solo a las distintas variedades lingüísticas, sino también "a las diferentes lenguas maternas existentes en el país". Asimismo, plantea la necesidad de incluir la formación plurilingüe a través de la enseñanza de segundas lenguas y lenguas extranjeras. Según Behares y Brovetto (2009:99), el término plurilingüe, en este caso, refiere a "la oferta de varias lenguas en el sistema educativo, lo que significa un cambio importante en las políticas educativas" ya que, a partir de la década de 1990, se dio prioridad al inglés en detrimento del francés y del italiano y fueron eliminados de la enseñanza obligatoria.

\section{El Programa CLE y el proyecto definitivo de Institucionalización}

En diciembre de 2012, el Consejo Educación Secundaria resolvió: "Aprobar el Proyecto definitivo de Institucionalización del Programa de Centro de Lenguas Extranjeras" (Circular $\mathrm{N}^{\circ}$ 3127). Dicho proyecto fue el "producto de conjugar lo establecido en el informe de la Comisión y el análisis crítico de las prácticas ya implementadas en el Programa CLE" (Moreno, 2012: [s.p.]). En el mismo, también se fundamenta la importancia del estudio de lenguas extranjeras, las funciones del Programa, su organigrama, reglamento de evaluación y pasaje de grado, entre otros temas.

\section{El Programa “Centros de Lenguas Extranjeras": su evolución y situación actual}

Estudiantes destinatarios.

$\mathrm{Al}$ momento de su creación, el Programa estaba dirigido exclusivamente a alumnos de $1^{\circ}$ a $3^{\circ}$ año de Ciclo Básico diurnos dependientes del Consejo de Educación Secundaria (en adelante CES) y del Consejo de Educación Técnico Profesional (en adelante CETP). A partir de 2009, se amplió la propuesta a estudiantes de Bachillerato, liceos extraedad y turnos nocturnos de CES y de CETP (CES, [s.f.]b; Comisión de Políticas Lingüísticas en la Educación, [s.f]).

Del Programa, se encuentran tácitamente excluidos los alumnos de Ciclo Básico y Bachillerato de liceos privados. 
Lenguas que se enseñan

El Programa fue creado para ofrecer la enseñanza de tres lenguas extranjeras: francés, italiano y portugués. En 1999, se agregó la enseñanza de alemán y, en 2014, de la lengua de señas uruguaya $(\mathrm{LSU})^{7}$.

Sin embargo, no todas las lenguas se enseñan en todos los centros, ni todos los centros reciben a todos los destinatarios de los cursos, y así surge de su convocatoria a inscripciones (cfr. CES, 2017).

\section{Organización de los cursos, niveles y certificaciones}

Los cursos para estudiantes de Ciclo Básico del CES y del CETP diurnos tienen una duración de tres años lectivos con la posibilidad de otro año de profundización. Los que están dirigidos a estudiantes de Bachillerato, turnos nocturnos y liceos extraedad del CES y de CETP tienen una duración de dos años, con opción a un año más de profundización ${ }^{8}$, si todavía están cursando Educación Media Básica o Superior (Moreno, 2012; Comisión de Políticas Lingüísticas en la Educación, [s.f.])

Todos los cursos se dictan a contraturno, en centros educativos denominados Centros de Lenguas Extranjeras (CLE). En la actualidad, existen 23 centros: seis en Montevideo y diecisiete en el interior del país ${ }^{9}$ (ANEP, 2014; 2015c).

Las clases tienen una duración de 90 minutos y una frecuencia de dos veces a la semana ${ }^{10}$. Se desarrollan dos módulos por año y, al finalizar cada uno de ellos, se realiza una evaluación a nivel nacional (CES, [s.f.]b; Moreno, 2012). Terminado el curso básico, los estudiantes reciben un diploma que acredita los conocimientos adquiridos de acuerdo con el Marco Común Europeo de Referencia para las Lenguas (Moreno, 2012). La realización del curso básico permitiría al alumno alcanzar un nivel básico o intermedio, según la lengua elegida.

\footnotetext{
${ }^{7}$ En CES ([s.f.]a:27) se señala que "por circunstancias particulares”, también se enseñó griego en Tacuarembó, y que en 2010 se iniciarían cursos de chino mandarín. En ningún otro documento se encontró información vinculada al dictado de esos cursos.

${ }^{8}$ A partir de 2011, en siete centros, se implementó un curso de profundización para aquellos estudiantes que desearan hacerlo (ANEP, 2014, 2015c; 2016).

${ }^{9}$ En Montevideo, los centros se ubican en los siguientes barrios: Centro, Flor de Maroñas, Prado, Cerrito de la Victoria, Peñarol y Pocitos. En el interior del país, se encuentran en: Artigas, Carmelo, Chuy, Durazno, Florida, Fray Bentos, Maldonado, Melo, Mercedes, Paysandú, Rivera, Salto, San José, Solymar, Tacuarembó, Treinta y Tres y Trinidad (CES, 2017).

${ }^{10}$ En CES ([s.f.]a:27) se indica que "tienen una carga horaria de cuatro horas semanales", por lo cual las clases no serían de hora y media sino de dos horas. En ANEP (2016:54) se señala que los cursos se dan "en régimen de dos sesiones semanales de dos horas".
} 
En el Proyecto de Presupuesto 2015-2019 se estableció que, entre los objetivos de la ANEP para el quinquenio, estaría la implementación de sistemas de acreditación de los aprendizajes a través de pruebas de validez internacional, para lo cual se buscaría la consolidación de las acreditaciones ofrecidas por la Alianza Francesa, el Instituto de Cultura Uruguayo-Brasileño, la Sociedad Dante Alighieri y el Instituto Goethe (ANEP, 2015b). En 2015, se proyectó ofrecer a 40 estudiantes de francés que hubieran obtenido el diploma de Curso Básico o de Nivel Intermedio en 2013 o 2014, la posibilidad de rendir el examen DELF ${ }^{11}$-niveles A2 y B1(ANEP, 2015a). En junio de 2016, se entregó a 31 alumnos los certificados correspondientes a dicho examen ${ }^{12}$. Sería interesante saber si todos los alumnos que rindieron el examen lo aprobaron; como así también cuántos estudiantes rindieron el examen en cada nivel. No se ha encontrado información publicada al respecto.

\section{El Programa “Centros de Lenguas Extranjeras” en cifras}

\section{Número de docentes}

Con respecto a la cantidad de profesores del Programa, solamente se encontró información correspondiente a los años 2007, 2010 y 2012.

En 2010, la cantidad total de profesores aumentó un 15\% con respecto a 2007, número que prácticamente se mantuvo en 2012, tal como se observa en el siguiente gráfico:

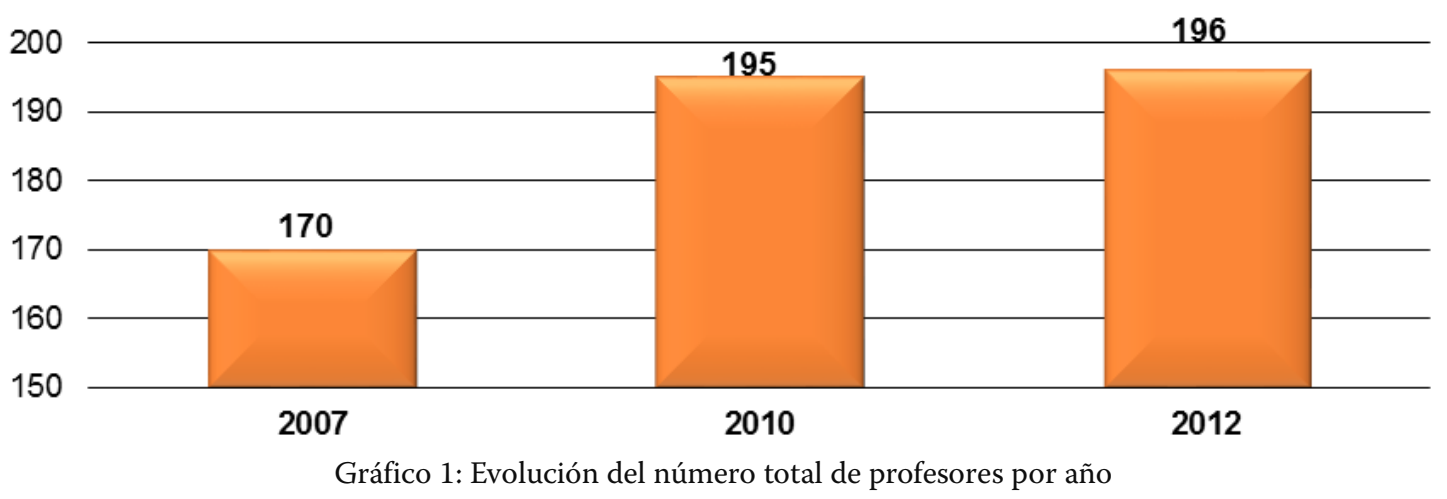

(Fuentes: datos de 2007 (ANEP, 2008b:411); datos de 2010 (CES, [s.f.]b:18); datos de 2012 (Comisión de Políticas Lingüísticas en la Educación, [s.f.]:[s. p.])

\footnotetext{
${ }^{11}$ El DELF (Diplôme d'etudes en langue française) es un certificado expedido por el Ministerio de Educación Nacional de Francia que acredita el nivel en la lengua francesa de candidatos extranjeros de países no francófonos.

${ }^{12}$ Publicado en <http://www.ces.edu.uy/index.php/centro-de-lenguas-extranjeras >
} 
En lo que refiere a la evolución del número de docentes de cada lengua, los datos fueron los siguientes:

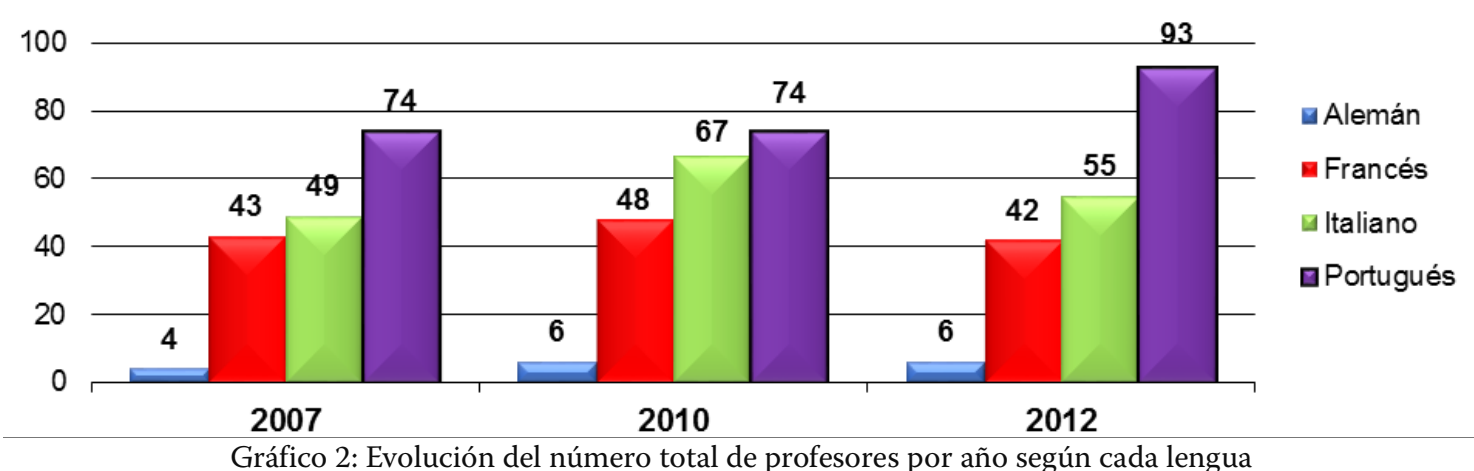

(Fuentes: datos de 2007 (ANEP, 2008b:411); datos de 2010 (CES, [s.f.]b:18); datos de 2012 (Comisión de Políticas Lingüísticas en la Educación, [s.f.]: [s. p.])

Se puede observar que, los profesores de francés aumentaron casi un 12\% de 2007 a 2010; sin embargo, ese porcentaje desaparece, ya que en 2012 la cantidad de profesores es casi la misma que en 2007. Por su parte, los docentes de italiano, en 2012, aumentaron un $12 \%$ con respecto a 2007, pero descendieron un 18\% con respecto a 2010. El número de profesores de portugués, entre 2007-2010, se mantuvo igual, pero tuvo un incremento del $28 \%$ en 2012. Por último, la cantidad de docentes de alemán aumentó en un 50\%, entre 2007 y 2010, número que se mantuvo en 2012.

Resulta llamativo el aumento del número de profesores de italiano y de francés en 2010 y su descenso en 2012, como así también, este mismo año, el aumento de casi un 30\% de los profesores de portugués. Surge la interrogante: ¿cuáles pudieron ser las causas de esos cambios?

Con respecto al aumento de profesores de portugués, de acuerdo con la Rendición de Cuentas del Ejercicio 2012, ese año comenzó a implementarse un Programa Diferencial de Portugués para los Centro de Lenguas Extranjeras de Frontera. El proyecto proponía "realizar programas diferenciados en portugués, tomando en cuenta la diversidad lingüística en el Uruguay" procurando "dotar a los CLE que se encuentran en el área de frontera con Brasil, de un programa diferencial, material didáctico y pruebas de evaluación para los cursos de portugués" (ANEP, 2013:76). Muy posiblemente aquí se encuentre la causa del aumento en el número de profesores de portugués, sin embargo, no se encontró información para confirmar esta hipótesis. 


\section{Número de alumnos según la lengua estudiada}

En lo que respecta al número de alumnos según la lengua estudiada, la lengua con mayor cantidad de alumnos ha sido el portugués, seguido por el italiano, el francés y el alemán.

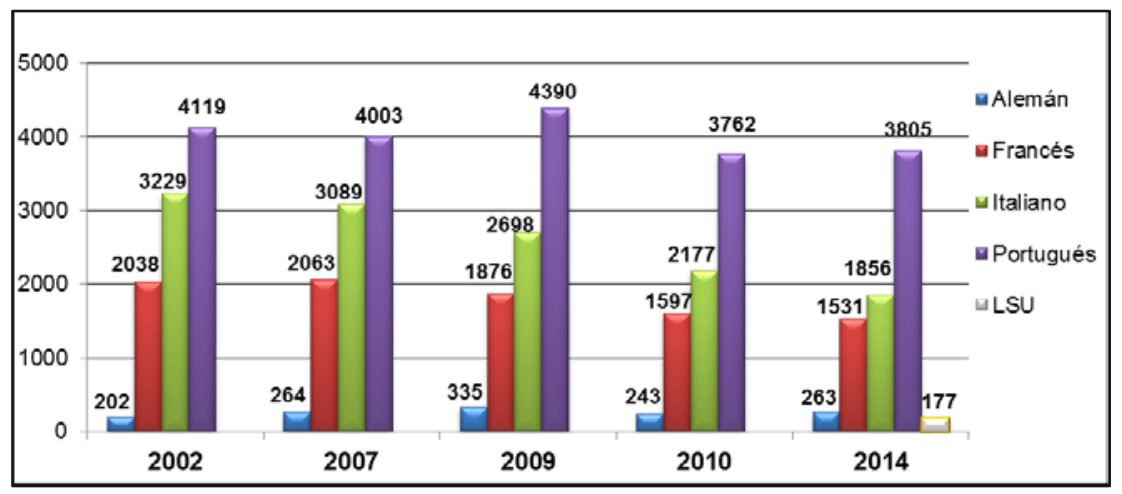

Gráfico 3: Evolución del número total de estudiantes por año según cada lengua

(Fuentes: datos de 2002 (Bertolotti, 2003:82); datos de 2007(ANEP, 2008a:246); datos de 2009 ${ }^{13}$ (CES, [s.f.]a: 27); datos de 2010 (CES, [s.f.]b:18); datos de 2014 (ANEP, 2015c:66))

Como puede observarse, desde 2002, la distribución de alumnos por lengua permanece inalterada. Tal distribución podría ser considerada el reflejo de las representaciones lingüísticas de las familias acerca de cuál es la mejor lengua extranjera para que sus hijos estudien como segunda opción ya que la primera es el inglés por ser de enseñanza obligatoria en la educación secundaria (Bertolotti, 2003). Con relación a la LSU, el único dato que surgió en la investigación corresponde a 2014 con lo cual no es posible realizar ningún análisis comparativo.

\section{Número de alumnos en Montevideo e interior del país}

En lo relativo al número de alumnos según se encuentren en Montevideo o en el interior de país, solamente se obtuvieron datos relativos a 2002 y 2010.

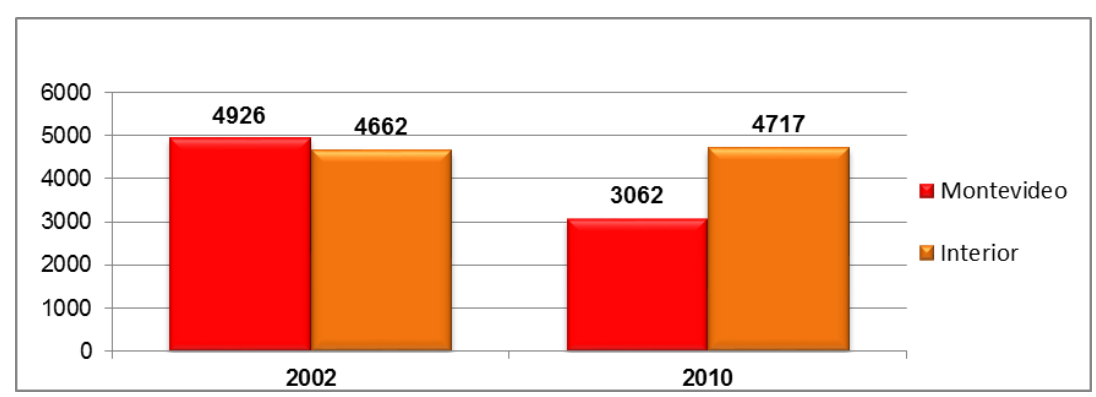

Gráfico 4: Evolución del número de alumnos en Montevideo e interior del país. (Fuentes: datos de 2002 (Bertolotti, 2003:82); datos de 2010 (CES, [s.f.]b:18))

\footnotetext{
${ }^{13}$ La misma información, pero con una diferencia de cuatro alumnos (uno más en alemán y tres más en francés), aparece en el informe de la Comisión de Políticas Lingüísticas en la Educación ([s.f.]), como correspondiente al año 2012. Se prefirió la información publicada en CES ([s.f.]a: 27) por encontrarse más acorde a la evolución que ha tenido el Programa CLE.
} 
En 2002, el alumnado de Montevideo superaba a los alumnos del interior, casi en un 6\%; en cambio, en 2010, el interior superó en más de un 50\% el número de estudiantes de Montevideo. Ante estos datos surge la siguiente pregunta: ¿Por qué se produce un descenso tan importante en los alumnos de Montevideo?

En lo que tiene que ver con cada lengua en particular, en Montevideo, tanto en 2002 como en 2010, a pesar del descenso en su número de alumnos, el portugués fue la lengua con mayor cantidad de estudiantes (Ver gráfico 5).

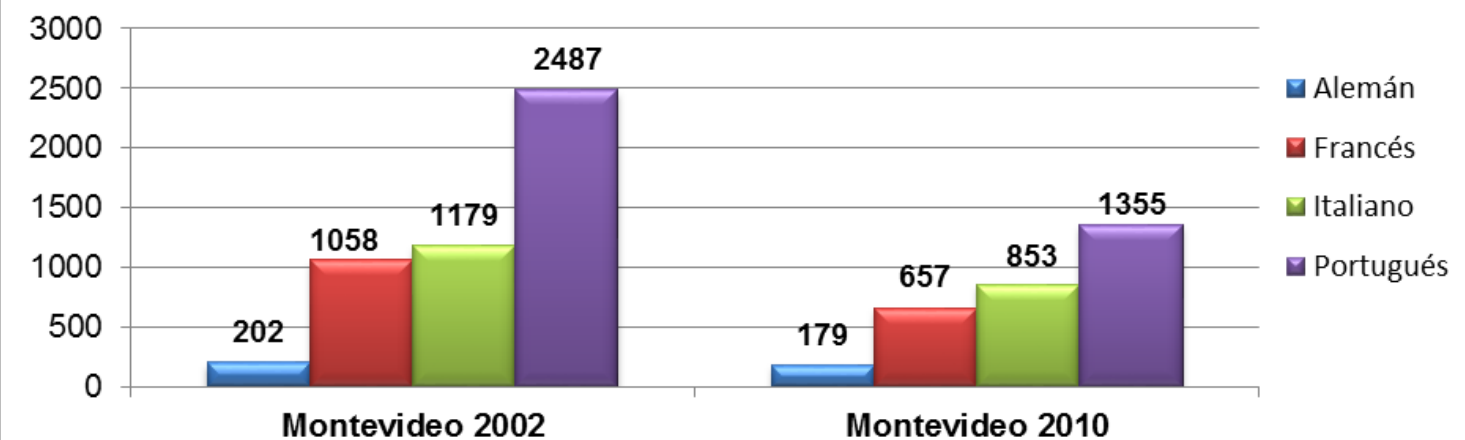

Gráfico 5: Evolución del número de alumnos de Montevideo según la lengua elegida

(Fuente: datos de 2002 (Bertolotti, 2003:82); datos de 2010 (CES, [s.f.]b:18))

En el interior, en cambio, en 2002 la lengua de preferencia fue el italiano; pero, en 2010, el portugués se ubicó en el primer lugar.

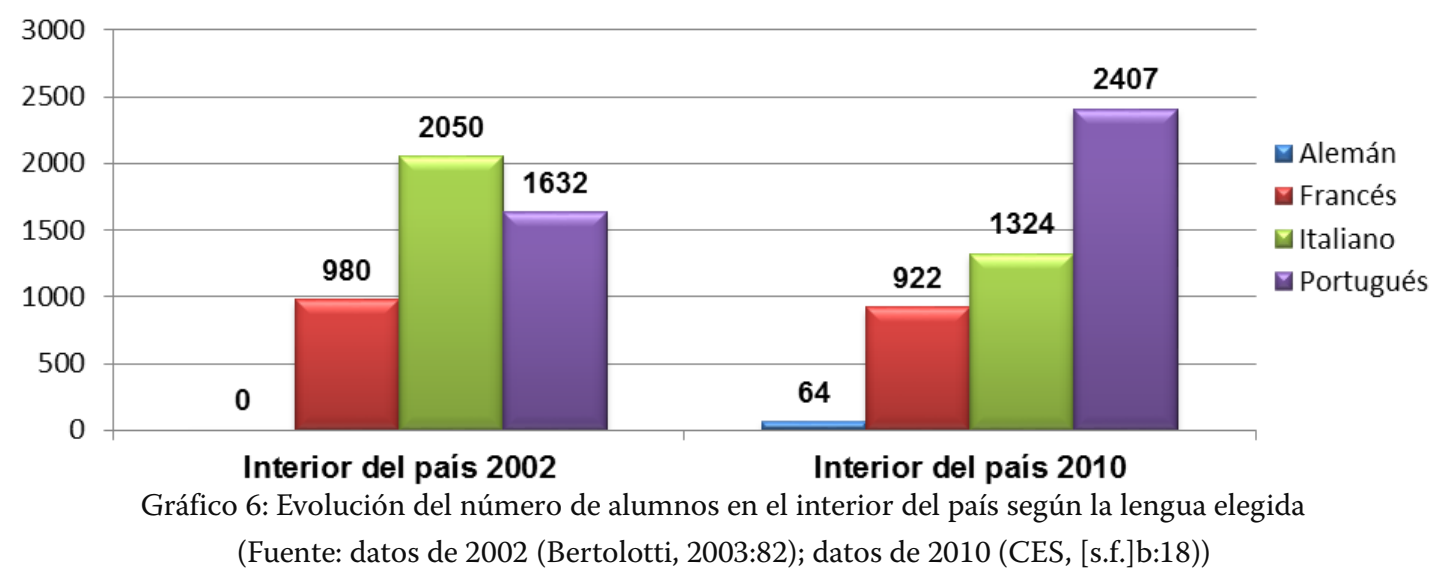

Asimismo, se presenta otra interrogante: ¿Cuáles fueron las causas que ocasionaron el descenso del alumnado de italiano y el correlativo incremento de alumnos de portugués, en el interior? No se ha encontrado información que permita contestar estas preguntas. 


\section{Número de alumnos inscriptos y total de destinatarios.}

Con respecto al total de alumnos inscriptos en el Programa, la evolución ha sido la siguiente:

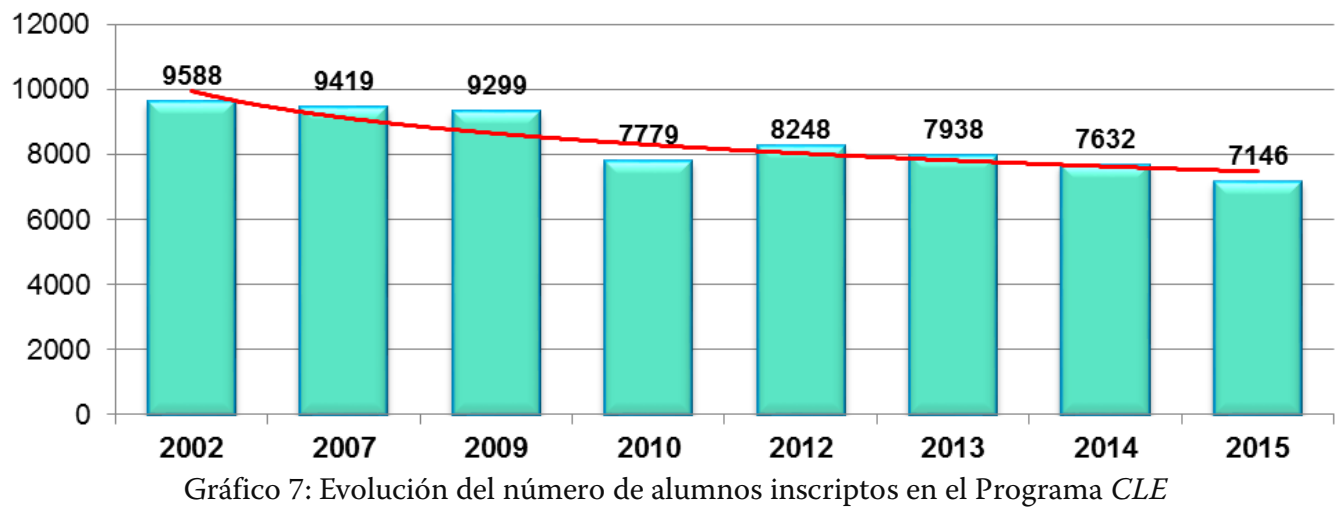

(Fuentes: datos de 2002 (Bertolotti, 2003:82); datos de 2007(ANEP, 2008a:246); datos de 2009 (CES, [s.f.]a: 27); datos de 2010 (CES, [s.f.]b:18); datos de 2012 (INEEd, 2014:76 y 80); datos de 2013 (ANEP, 2014:74); datos de 2014 (ANEP, 2015c:66); datos de 2015 (ANEP (2016:54).

Desde $2002^{14}$ se puede apreciar un definido y constante descenso en el número de estudiantes del Programa CLE .

Como se dijo anteriormente, la oferta del Programa CLE estaba dirigida exclusivamente a los alumnos de Ciclo Básico Diurno de CES y CETP. A partir de 2009, se amplió la oferta de los cursos a los estudiantes de Bachillerato (CES y CETP), turnos nocturnos y liceo extraedad. En el siguiente gráfico se observa de manera comparativa la evolución del número de alumnos destinatarios y del número de inscriptos en el Programa CLE, entre 2002 y 2015.

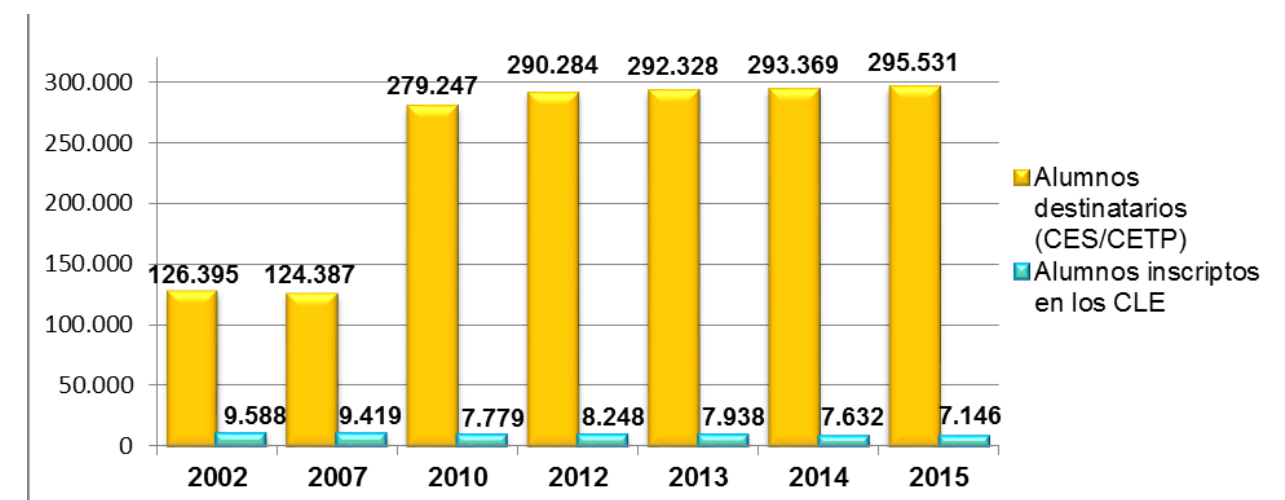

Gráfico 8: Evolución del número de alumnos destinatarios y de alumnos inscriptos en el Programa CLE (Fuentes: datos de 2002 y 2007 (MEC, 2013[CD-ROM]); datos de 2010, 2012,2013, 2014 y 2015 (MEC, 2015))

\footnotetext{
${ }^{14}$ No se encontró información anterior a esa fecha.
} 
Según los datos publicados en MEC (2013 y 2015), los alumnos destinatarios de los cursos hasta 2009 estaba en el entorno de los 125.000 estudiantes ya que solamente incluía a los estudiantes de Ciclo Básico diurnos de CES y CETP, mientras que el número de los alumnos efectivamente inscriptos en el Programa promediaba los 9.500 alumnos. A partir de $2010^{15}$, el número de destinatarios se incrementó a más de 280.000 estudiantes; sin embargo, el número de alumnos inscriptos descendió a una media de 7.750 alumnos.

En otras palabras, la cantidad de alumnos que participaban de los CLE entre 2002-2007 representaba el 7,6 \% del universo de destinatarios. A partir de 2010, ese porcentaje pasó a ser menor al 3\% del total de estudiantes con derecho a los cursos.

Con respecto al porcentaje de alumnos inscriptos, también surgen interrogantes: ¿cuántos de los alumnos inscriptos pertenecen a CES y cuántos a CETP?, ¿cuántos aprueban los cursos cada año?, ¿cuántos realizan y aprueban el programa en su totalidad?, ¿cuántos realizan el curso de profundización? No se ha encontrado información publicada al respecto ${ }^{16}$.

Por otro lado, los datos analizados, llevan al planteo de nuevas preguntas: ¿Cuál es la razón por la cual más del 95\% de los alumnos no se adhiera al Programa? ¿el Estado uruguayo no ha realizado una propaganda y/o publicidad suficientemente atractiva como para construir representaciones lingüísticas positivas hacia el aprendizaje de lenguas extranjeras? ¿será que el Programa no tiene una oferta de cursos según las necesidades y aspiraciones de los destinatarios?

\section{Algunas reflexiones finales a modo de síntesis.}

La enseñanza de lenguas extranjeras estuvo tradicionalmente presente en los planes de estudio de Educación Secundaria. Las lenguas a las que se dio prioridad fueron francés, inglés e italiano, las cuales integraron el currículo obligatorio con diseños programáticos y cargas horarias que fueron variando en el tiempo; el portugués nunca estuvo incluido.

\footnotetext{
${ }^{15}$ Los datos que corresponden a 2009 no se tuvieron en cuenta por entender que, por ser un año de transición, no serían representativos.

16 Únicamente se encontró información sobre los alumnos promovidos en 2006: en total (3.149) y según cada lengua (87 en alemán, 801 en francés, 1.217 en italiano y 1.044 en portugués) (ANEP, 2008a:246). Al no encontrarse información con relación al número total de inscriptos ese año, ni al número de promovidos en ningún otro año, no se ha podido realizar ningún análisis comparativo. Sin embargo, si se tiene en cuenta que, de acuerdo con los datos obtenidos, entre 2002 y 2009, la media aproximada de la cantidad total de alumnos fue 9.435, el porcentaje de alumnos aprobados estaría situado en el entorno del 33\%, aunque este dato no es más que una referencia, y no permite llegar a conclusiones definitivas.
} 
En la década del 90, se estableció que, a nivel de la educación media, el inglés fuera la única lengua extranjera de enseñanza obligatoria, y se creó el Programa "Centro de Lenguas Extranjeras" para impartir cursos opcionales y extracurriculares de francés, italiano y portugués.

Actualmente, el Programa CLE ofrece cursos de francés, italiano, portugués, alemán y LSU a alumnos del ámbito público de CES y CETP. Según los datos recabados, la lengua con mayor alumnado, tanto en Montevideo como en el interior del país, ha sido el portugués, seguida por el italiano, el francés y el alemán. La distribución de las lenguas según el número de alumnos ha sido la misma desde el año 2002.

En lo que refiere a cada lengua en particular, el hecho de que el portugués sea la lengua con mayor cantidad de estudiantes podría significar que su estudio es considerado la mejor opción, lo cual es llamativo teniendo en cuenta que las políticas lingüísticas aplicadas por el Estado uruguayo hasta, al menos, la década de 1990, han buscado generar representaciones lingüísticas negativas hacia esa lengua con el objetivo de fortalecer las fronteras lingüísticas y culturales del país. Por su parte, el italiano se encuentra en segundo lugar a pesar de su relevancia por el gran porcentaje de población de origen italiano, y de ser la lengua que ha perdido mayor cantidad de estudiantes. Con respecto al francés, a pesar de que ha sido la lengua que ha integrado históricamente la mayor parte de los planes de estudio, se encuentra en el tercer lugar en el número de participantes; no obstante, es la única -hasta el momento- en la que se puede obtener una certificación internacional. En relación con el alemán, a pesar de encontrarse en el último lugar en número de estudiantes y, de que sus cursos no se ofrecen en todos los centros (cfr. CES, 2017), es la única lengua que ha presentado un crecimiento constante.

Sin embargo, no es posible concluir que el orden de las lenguas según el número de alumnos sea necesariamente reflejo de sus verdaderas preferencias. Estos datos solamente podrían ser tomados como expresión de sus intereses si todos los estudiantes tuvieran las mismas oportunidades de acceder a todos los cursos en todos los centros. No obstante, la oferta no es similar en todas las sedes (cfr. CES, 2017).

Durante el período 2002-2015, se han inscripto en el Programa CLE un promedio 8.250 estudiantes. Sin embargo, a partir de 2002, existe un constante descenso en el número de alumnos, siendo el 2015 el año que registró menos inscripciones.

A pesar de que en 2009 fue ampliada la oferta de los cursos a casi todas las áreas de CES y CETP, el número de inscriptos continúa descendiendo al punto que el porcentaje de alumnos no alcanza el 3\% del total de estudiantes a los cuales está dirigido el Programa. 
A esto se debe agregar que, sin encontrarse una razón explícita, se excluyeron del Programa a todos los alumnos de la enseñanza privada, por lo cual, alrededor de 42.000 estudiantes no tienen la posibilidad de acceder a los cursos. De esta manera, se deja fuera del Programa aproximadamente al $12 \%$ de la población estudiantil.

Ante el escaso el número de estudiantes que aprovecha los cursos del Programa CLE sumado al porcentaje de alumnos que no puede participar del mismo, surge la siguiente pregunta: ¿Se podrá alcanzar la meta propuesta para el año 2030 ?

Para finalizar, el estudio aquí presentado ha analizado el Programa CLE con la profundidad que ha permitido la información disponible. Se debe señalar que, a pesar de los resultados obtenidos, el Programa CLE es una muy buena iniciativa llamada a cumplir un papel fundamental en el logro de las metas que el Estado uruguayo se ha propuesto alcanzar. Por lo que, a más de 20 años de su creación y teniendo en cuenta los cambios sociales y tecnológicos que han sucedido en este tiempo, deberían explorarse nuevos caminos que le permitan lograr el máximo cumplimiento de sus cometidos en orden a alcanzar así los objetivos planteados.

\section{Referencias bibliográficas}

Araujo, O. (1906). Historia de la escuela uruguaya. Tomo II. Montevideo: Dornaleche y Reyes Editores.

Administración Nacional de Educación Pública (2008a). Documentos de la Comisión de Políticas Lingüísticas en la Educación Pública (2006-2007). Disponible en: $<$ http://www.anep.edu.uy/plinguisticas/phocadownload/documentos/comisionpoliticaslinguisticaseducacion\%20publica.pdf $>$ [Consultado el 23 de noviembre de 2015]

Administración Nacional de Educación Pública (2008b). Historia de Educación Secundaria 1935-2008. Montevideo: Tarma.

Administración Nacional de Educación Pública (2012). Rendición de cuentas 2011. Disponible en: $\quad<$ http://www.anep.edu.uy/anep/phocadownload/Presupuestosyrendiciones/RCBEP-2011/RCBEP-2011.pdf > [Consultado el 30 de noviembre de 2015]

Administración Nacional de Educación Pública (2013). Rendición de cuentas 2012 [pdf]. Disponible en: <http://www.anep.edu.uy/anep/phocadownload/Presupuestosyrendiciones/RCBEP-2012/RCBEP-2012.pdf $>$ [Consultado el 30 de noviembre de 2015]

Administración Nacional de Educación Pública (2014). Relevamiento de Programas Centrales y de los Consejos Desconcentrados [pdf]. Disponible en: 
<http://www.anep.edu.uy/anep/phocadownload/dspe/Oferta\%20educativa_Programas_Proyectos2014.pdf> [Consultado el 2 de marzo de 2016]

Administración Nacional de Educación Pública (2015a). Proyectos 2015. Certificación internacional en lengua francesa para estudiantes egresados del programa CLE [pdf]. Disponible en: <https://www.ces.edu.uy/ces/images/stories/2015/junio/Proyectos_2015_PCLE-1.pdf> [Consultado el 7 de febrero de 2016]

Administración Nacional de Educación Pública (2015b). Proyecto de presupuesto. Período 2015-2019. Exposición de motivos, 1 [pdf]. Disponible en: $<$ http://www.anep.edu.uy/anep/phocadownload/Presupuestosyrendiciones/ProyectodePresupuesto_Periodo_2015-2019/Tomo\%201\%20-\%20Еxposici\%C3\%B3n\%20de\%20Motivos\%20ANEP.pdf> [Consultado el 30 de noviembre de 2015]

Administración Nacional de Educación Pública (2015c). Relevamiento de Programas Centrales $y$ de los Consejos Desconcentrados [pdf]. Disponible en: < $\underline{\text { http://pcentra- }}$ les.anep.edu.uy/phocadownload/2015/2015_Informe_Final_ch.pdf> [Consultado el 22 de diciembre de 2015]

Administración Nacional de Educación Pública (2016). Relevamiento de iniciativas de políticas y proyectos educativos -2016- [pdf]. Disponible en: <http://pcentrales.anep.edu.uy/phocadownload/2016/Informe2016.pdf> [Consultado el 13 de setiembre de 2017]

Barrios, G. (1996). Planificación lingüística e integración regional: el Uruguay y la zona de frontera. En Menine Trindade, A. y Behares, L. (orgs.) Fronteiras, educaçaó, integraçaõ. Santa Maria: Pallotti.

Barrios, G. (2011). El tratamiento de la diversidad lingüística en la educación uruguaya (20062008). Letras, 21 (42); p.15.

Barrios, G. (2013). Language diversity and national unity in the history of Uruguay. En Del Valle, J. (ed.) A Political History of Spanish: The Making of a Language. Nueva York: Cambridge University Press.

Barrios, G. (2015). Política lingüística y dictadura militar en Uruguay (1973-1985): los informes institucionales sobre la situación lingüística fronteriza. Estudios de Lingüística del Español, 36, p. 527. Disponible en <http://infoling.org/elies/36/elies36-20.pdf> [Consultado el 6 de febrero de 2016] 
Behares, L. y Brovetto, C. (2009). Referencias al lenguaje en las leyes de educación de Uruguay Actas del IV Encontro Internacional de Pesquisadores de Políticas Linguísticas, (pp. 95102). Santa María: UFSM-AUGM

Bertolotti, V. (2003). Uruguay. En Unión Latina, Relevamiento de la enseñanza de lenguas romances en el cono sur. Montevideo: FHCE, Udelar.

Brovetto, C. (2010). Las políticas lingüísticas de la ANEP: balance y perspectivas. En Yarzábal, L. (coord.), Una transformación en marcha. Políticas instrumentadas por el CODICEN -2005-2009-. Montevideo: ANEP-CODICEN.

Bralich, J. (2013). Una historia de la educación uruguaya [recurso electrónico] [s.l.]:[s.n.]. Disponible en: Facultad de Humanidades y Ciencias de la Educación. Ubicación CD137.

Circular N 3127 [6 de diciembre de 2012]. Consejo de Educación Secundaria. Disponible en: $<$ http://www.ces.edu.uy/index.php/circulares11/5410-exp1293012-circularno3127mlp>

Consejo de Educación Secundaria ([s.f.]a). Memoria CES. 2005-2010 [pdf]. Disponible en:


brero de 2016]

Consejo de Educación Secundaria ([s.f.]b). Programa 'Centros de Lenguas Extranjeras - Presentación-' [pdf]. Disponible en: <https://www.ces.edu.uy/ces/images/stories/CLE/presentacincle.pdf> [Consultado el 17 de noviembre de 2015]

Comisión de Políticas Lingüísticas en la Educación y Dirección de Investigación, Evaluación y Estadística ([s.f.]) Ampliación de la enseñanza de lenguas extranjeras. Propuesta integral para la educación pública (2012-2013) [pdf]. Disponible en: $<$ http://www.anep.edu.uy/plinguisticas/phocadownload/proyectos2013/proyectos\%202012-13\%20.pdf> [Consultado el 6 de febrero de 2016]

Consejo de Educación Secundaria (2016). Monitor Educativo Liceal. Principales resultados 2015 [pdf]. Disponible en: <http://www.anep.edu.uy/anep/phocadownload/Noticias_Doc/2016/principales\%20resultadosces\%20monitor\%202015.pdf> [Consultado el 19 de agosto de 2017]

Consejo de Educación Secundaria (2017). Requisitos para inscripciones en los CLE. [pdf] Disponible en: <https://www.ces.edu.uy/files/2017/news/Inscripciones_pag_Web.pdf> [Consultado el 19 de agosto de 2017] 
Consejo de Educación Técnico Profesional ([s.f]) Informe Matrícula 2015. Programa Planeamiento Educativo 2015 [pdf]. Disponible en: <https://planeamientoeducativo.utu.edu.uy/portal/images/Estadistica/Estadistica/Matricula/Informe-2015.pdf> [Consultado el 19 de agosto de 2017]

Instituto Nacional de Evaluación Educativa (2014). Informe Nacional sobre la Educación en Uruguay 2014. Montevideo: INEEd.

La Paz Barbarich, E. (2012). Imperialismo lingüístico: el caso del inglés en la educación secundaria uruguaya (1941-2003). Lingüística, 27, p.168. Disponible en:

$<$ http://www.scielo.edu.uy/pdf/ling/v27n1/v27n1a08.pdf> [Consultado el 6 de febrero de 2016]

Ley 18.437 - Ley General de Educación-. [Publicada en Diario Oficial el 16 enero de 2009]. Disponible en: <http://www.impo.com.uy/bases/leyes/18437-2008>

Nahum, B. (1994). Manual de historia del Uruguay (1830-1903). Montevideo: Ediciones de la Banda Oriental

Mercado Común del Sur. (1991). Protocolo de Intenciones [pdf]. Disponible en: $<\underline{\text { http://repo- }}$ sitorio. educacion.gov.ar/dspace/bitstream/handle/123456789/66160/protocolo_intenciones.pdf?sequence $=1>$ [Consultado7 de noviembre de 2016]

Ministerio de Educación y Cultura. Dirección de Educación (2013). Anuario Estadístico de Educación 2012, [CD-ROM]. Montevideo: Rojo.

Ministerio de Educación y Cultura. -Dirección de Educación. Área de investigación y estadística- (2014). A 140 años de la Educación del pueblo: Aportes para la reflexión sobre la educación en Uruguay. Montevideo: MEC

Ministerio de Educación y Cultura. -Dirección de Educación- (2015). Anuario Estadístico de Educación 2014. Disponible en: <http://www.mec.gub.uy/innovaportal/v/11078/5/mecweb/publicaciones ?3colid=927> [Consultado el 6 de febrero de 2016].

Moreno, A. (2012). Proyecto definitivo de Institucionalización del Programa de Centro de Lenguas Extranjeras [pdf]. Disponible en: $<$ http://www.ces.edu.uy/ces/images/comunicaciones/Institucionalizacin PCLE.pdf $>$ [Consultado el 23 de noviembre de 2015].

Oroño, M. (2011). La escuela pública uruguaya y el idioma nacional en dos períodos históricos: fines del siglo XIX y mediados del siglo XX. Letras, 42, p. 217. Disponible en: 
<https://periodicos.ufsm.br/letras/article/viewFile/12182/7576> [Consultado el 11 de agosto de 2016].

Oroño, M. (2012). La escuela y la lengua en la construcción discursiva de la identidad nacional: los libros de lectura usados en la escuela uruguaya en el último cuarto del siglo XIX. En Masello, L. (dir.) Lenguas en la región. Enseñanza e investigación para la integración desde la universidad (pp. 77-95). Montevideo: FHCE, Udelar.

Oroño, M. (2016). La escuela en la construcción de las fronteras culturales y lingüísticas en el Uruguay de fines del siglo XIX. Páginas de Educación, 9(1), p.146. Disponible en: $<$ http://www.scielo.edu.uy/pdf/pe/v9n1/v9n1a06.pdf> [Consultado el 11 de agosto de 2016].

Reyes Abadie, W. y Vázquez Romero, A. (2000a). Crónica General del Uruguay. Tomo IV -El Estado Oriental-. Montevideo: Ediciones de la Banda Oriental.

Reyes Abadie, W. y Vázquez Romero, A. (2000b). Crónica General del Uruguay. Tomo V -La Modernización-. Montevideo: Ediciones de la Banda Oriental.

Traversoni, A. (1987). Historia del Uruguay y de América. Tomo II. Montevideo: Kapeluz. 